Pacific Journal of Mathematics

DIVISOR CLASSES IN PSEUDO GALOIS EXTENSIONS 


\section{DIVISOR CLASSES IN PSEUDO GALOIS EXTENSIONS}

\section{William C. WATERHOUSE}

Let $R$ be a Krull domain with fraction field $K$. Let $L$ be a finite extension of $K$, and let $S$ be the integral closure of $R$ in $L$; then $S$ is also a Krull domain. Let $\mathscr{P}(R, S)$ be the group of divisor classes in $R$ becoming principal in $S$. Suppose there is a group scheme (or Hopf algebra) acting on $S$ with fixed ring $R$. Then there is a cohomology group which contains $\mathscr{P}(R, S)$ and equals it if the action is Galois at each minimal prime. This generalizes and unifies some results of Samuel.

1. Definition of the cohomology group. Let $R, K, L$ and $S$ be as above. Let $H$ be a cocommutative Hopf algebra over $R$, with $\delta, \varepsilon$, and $\rho$ its comultiplication, counit, and coinverse. One calls $S$ an $H$ module algebra [9, p. 207] if it has an $H$-module structure such that $h \cdot 1=\varepsilon(h)$ and $h \cdot\left(s s^{\prime}\right)=\sum\left(h_{i} \cdot s\right)\left(h_{i}^{\prime} \cdot s^{\prime}\right)$ where $\delta(h)=\sum h_{i} \otimes h_{i}^{\prime}$. We say that $R$ is the fixed ring in $S$ if

$$
R=\{s \in S \mid h \cdot s=\varepsilon(h) s \text { for all } h \in H\} \text {. }
$$

In this case $L$ is naturally an $H$-module algebra with fixed ring $K$.

Suppose now $S$ is an $H$-module algebra with fixed ring $R$, and consider the set

$$
\left\{b \in L^{*} \mid b^{-1}(h \cdot b) \in S \text { for all } h \in H\right\} .
$$

This is a group under multiplication: if $b$ and $c$ are in it, we have

$$
(b c)^{-1} h \cdot(b c)=\sum\left(b^{-1} h_{i} \cdot b\right)\left(c^{-1} h_{i}^{\prime} \cdot c\right)
$$

and

$$
\left(h \cdot b^{-1}\right) b=\sum h_{i} \cdot\left[b^{-1}\left(\rho h_{i}^{\prime}\right) \cdot b\right]
$$

It contains $S^{*}$ and $K^{*}$ as subgroups. We write $H^{0}\left(H, L^{*} / S^{*}\right)$ for its quotient by $S^{*}$, and $\mathbb{Q}^{2}(H, S)$ for the quotient by $S^{*} K^{*}$. Note that $h \mapsto b^{-1} h \cdot b$ defines a function $H \rightarrow S$; it is easy to check that $b$ and $c$ give the same function if and only if $b c^{-1}$ is in the fixed ring $K$, and hence we can also view $Q^{2}$ as these functions modulo the functions coming from units $b \in S^{*}$.

Proposition 1. Assume $S$ is an $H$-module algebra with fixed ring R. Then there is a canonical injection

$$
\mathscr{P}(R, S) \rightarrow \mathbb{Q}^{P}(H, S)
$$


Proof. Let $D$ be a divisorial ideal of $R$ with $\operatorname{div}(D S)$ principal, say $=b S$. Let $P$ be a minimal prime of $R$, and choose $r \in K$ with $\operatorname{ord}_{P} r=\operatorname{ord}_{P} D$; then $b S_{P}=r S_{P}$. For any $h \in H$ we have

$$
h \cdot b \in h \cdot r S_{P}=r h \cdot S_{P} \cong r S_{P}=b S_{P},
$$

and hence $b^{-1} h \cdot b \in \bigcap_{P} S_{P}=S$. The element $b$ is well determined up to multiplication by an element of $S^{*}$, and thus we have a map (obviously a homomorphism) from such ideals $D$ to $H^{0}\left(H, L^{*} / S^{*}\right)$. Since $\operatorname{div}(D S)=S$ implies $D=R$, the map is injective. Divide now by $K^{*}$ in both places.

One can define [9] a sequence of cohomology groups $H^{i}\left(H, S^{*}\right)$. In that theory $H^{1}\left(H, S^{*}\right)$ consists of certain equivalence classes of functions $H \rightarrow S$; it maps naturally to $H^{1}\left(H, L^{*}\right)$, and the kernel comprises functions of the form $h \mapsto b^{-1} h \cdot b$. Under our hypotheses also $H^{0}\left(H, S^{*}\right)=R^{*}$ and $H^{0}\left(H, L^{*}\right)=K^{*}$. Thus our group $H^{0}\left(H, L^{*} / S^{*}\right)$ fits into an exact sequence, and $Q^{(}(H, S)$ is its image in $H^{1}\left(H, S^{*}\right)$.

Suppose that $G$ is a group, $H=R[G]$. To make $S$ an $H$-module algebra is simply to let $G$ act as $R$-algebra automorphisms of $S$. The definition of fixed ring is then the usual one, and $H^{\circ}\left(H, L^{*} / S^{*}\right)$ is the subset of $L^{*} / S^{*}$ fixed by $G$. In addition [9, p. 211], the cohomology $H^{1}\left(H, S^{*}\right)$ is naturally isomorphic to $H^{1}\left(G, S^{*}\right)$.

Suppose on the other hand that $H$ is the polynomial ring $R[X]$, with $\delta(X)=X \otimes 1+1 \otimes X, \varepsilon(X)=0$, and $\rho(X)=-X$. Then an $H$ module algebra structure is given by an $R$-linear derivation $D: S \rightarrow S$ (where $D s=X \cdot s$ ). The fixed ring is $\{s \mid D s=0\}$. The values $b^{-1} h \cdot b$ are determined by $b^{-1} D b$, and all lie in $S$ if this one does; hence $\mathbb{Q}(H, S)$ can be identified with the logarithmic derivatives $D b / b$ lying in $S$, modulo the logarithmic derivatives of elements of $S^{*}$. Thus it is the group introduced by Samuel in [7, p. 86], and our formalism unifies the two separate theories he presents. We could similarly take a finite set of derivations, let $H$ be an enveloping algebra for them, and get the group used in [10] and [11]. (The paper [11] contains a different connection between Samuel's group and cohomology, but it appears to be ad hos rather than natural.)

Suppose that $H$ is finite, i.e., a finitely generated projective $R$ module; this is the most important case. Let $A=\operatorname{Hom}(H, R)$ be the linear dual, a commutative Hopf algebra. Making $S$ an $H$-module algebra is then the same thing as giving an algebra homomorphism $\sigma: S \rightarrow A \otimes_{R} S$ suitably compatible with the comultiplication and counit of $A$ (cf. [5, p. 33]); in geometric language, this is an action of the finite group scheme Spec $A$ on Spec $S$ over Spec $R$. In these terms

$$
\mathbb{Q}(H, S)=\left\{\sigma(b) b^{-1} \mid b \in L^{*}, \sigma(b) b^{-1} \in(A \otimes S)^{*}\right\} / S^{*} ;
$$


the group $H^{1}\left(H, S^{*}\right)$ is the quotient by $S^{*}$ of the equalizer of two homomorphisms from $(A \otimes S)^{*}$ to $(A \otimes A \otimes S)^{*}$, and so on. One could phrase all the results equally well in terms of $A$, and I have used $H$ only because it is closer to the language used in the literature.

2. Conditions for isomorphism. Assume $S$ is an $H$-module algebra with $H$ finite. We say that $S$ with this structure is Galois if the following equivalent conditions hold [5, p. 66]:

(I) $S$ is a finitely generated projective $R$-module, and the map $H \otimes_{R} S \rightarrow \operatorname{End}_{R} S$ given by $h \otimes s_{0} \mapsto\left[s \mapsto s_{0} h \cdot s\right]$ is an $R$-module isomorphism.

(II) $S$ is a faithfully flat $R$-module, and

$$
\left(\sigma, 1 \otimes i d_{S}\right): S \otimes_{R} S \longrightarrow A \otimes_{R} S
$$

is an $R$-algebra isomorphism. In geometric language, this says [6, p. 27] that Spec $S$ is a principal homogeneous space for Spec $A$. It implies that $R$ is the fixed ring.

Proposition 2. Suppose $H$ is finite. If $L$ is Galois as an $H \otimes_{R} K$-module algebra, then

$$
\mathbb{Q}(H, S)=H^{1}\left(H, S^{*}\right) \text {. }
$$

Proof. This will follow if we show that $H^{1}\left(H, L^{*}\right)=0$. But it is easy to see from the definition (cf. end of $\S 1$ ) that this group equals $H^{1}\left(H \otimes K, L^{*}\right)$, which since the structure is Galois equals [9, p. 219] the Amitsur cohomology $H^{1}\left(L / K, \mathbf{G}_{m}\right)$; this is 0 by the generalized Hilbert Theorem 90 [1, p. 96 or 6, p. 15].

Theorem 1. Assume $S$ is an H-module algebra with $H$ finite. The following are equivalent:

(i) For all minimal primes $P$ of $R$, the $H_{P}$-structure on $S_{P}$ is Galois.

(ii) $R$ is the fixed ring, and for all minimal primes $P$ of $R$ the $H_{P} / P H_{P}$-structure on $S_{P} / P S_{P}$ is Galois.

(iii) $R$ is the fixed ring, and for all minimal primes $P$ of $R$ the map

$$
S_{P} / P S_{P} \otimes S_{P} / P S_{P} \rightarrow A_{P} / P A_{P} \otimes S_{P} / P S_{P}
$$

is an isomorphism.

(iv) The map $S \otimes S \rightarrow A \otimes S$ is a pseudo-isomorphism [in the sense that its $R$-module kernel and cokernel vanish when localized to any minimal prime].

These conditions imply

(v) $R$ is the fixed ring, and the map $H \otimes S \rightarrow \operatorname{End}_{R} S$ is a 
pseudo-isomorphism; they are equivalent to it if we assume either $R$ Noetherian or $S$ a finitely generated $R$-module.

Proof. If (i) holds then $R$ is the fixed ring because $R=\bigcap R_{P}$. Obviously (i) is equivalent to (iv), which implies (iii); and (iii) is equivalent to (ii) since $A_{P} / P A_{P}$ is the $R_{P} / P R_{P}$-dual of $H_{P} / P H_{P}$. If we now assume (ii) we have $\operatorname{dim} H_{P} / P H_{P}=\operatorname{dim} S_{P} / P S_{P}$. We know [3, p. 147] that the latter is $\leqq|L: K|$, with equality only if $S_{P}$ is a free $R_{P}$-module. But we also know that $K$ is the fixed ring in $L$, and it follows [9, p. 219] that $\operatorname{dim} H_{P} / P H_{P}=\operatorname{dim}_{K} H \otimes K \geqq|L: K|$. Hence we conclude that $S_{P}$ is free. But then the map $S_{P} \otimes S_{P} \rightarrow A_{P} \otimes S_{P}$, which is an isomorphism modulo $P$, is an actual isomorphism by Nakayama's lemma.

As for (v), we have the diagram

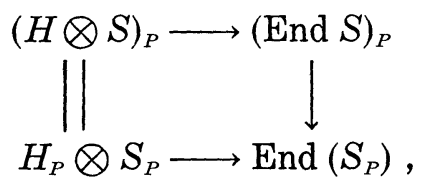

where we know that the arrow on the right is injective for any $S$ and surjective if $S$ is finitely generated [4, p. 49]. If we assume (i) we have an isomorphism on the bottom, and hence we must have an isomorphism on the top; if $S$ is finitely generated we can reverse the implication.

We claim now that $\left(\operatorname{End}_{R} S\right) \otimes K=\operatorname{End}_{K} L$ if and only if $S$ is an $R$-lattice in $L$. Indeed, if $S$ is an $R$-lattice, then $\operatorname{End}_{R} S$ is an $R$-lattice in $\operatorname{End}_{K} L$ by $\left[4\right.$, p. 45]. For the converse let $1=s_{1}, s_{2}, \cdots, s_{n}$ be a basis of $L$, and consider the maps $\varphi_{i}: \sum \alpha_{j} s_{j} \mapsto\left(\alpha_{i}\right) 1$. If $\operatorname{End}_{R} S$ is sufficiently large there is a $0 \neq r \in R$ such that the $r \varphi_{i}$ map $S$ into $S$, and then $S \subseteq(1 / r)\left(R s_{1}+\cdots+R s_{n}\right)$.

Now assume (v) with $R$ Noetherian. The fact that $K$ is the fixed ring implies again that $\operatorname{rank}(H) \geqq|L: K|$, so by dimension count (End $S$ ) $\otimes K$ is all of $\operatorname{End}_{K} L$. Then $S$ is an $R$-lattice, hence finitely generated, and the earlier argument applies.

If the conditions of the theorem hold, we say that $S$ with its $H$ structure is pseudo-Galois. One result of the proof deserves to be noted:

Porism. If $R$ is Noetherian and $S$ is pseudo-Galois, then $S$ is finitely generated over $R$.

THEOREM 2. Assume that $S$ is a pseudo-Galois H-module algebra. Then

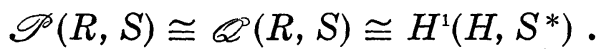


Proof. We know (by further localization) that $L$ is Galois for $H \otimes K$, so the second isomorphism is just Proposition 2. Take now a $b \in L^{*}$ with $h \cdot b \in b S$ for all $h \in H$; we must prove that $b S$ comes from a divisor of $R$. This is a local statement, so we may assume that $R$ is a discrete valuation ring and $S$ is Galois. It follows then that $b S$ is mapped to itself by all elements of $\operatorname{End}_{R} S$. Choose a basis $s_{1}, \cdots, s_{n}$ of $S$ and elements $r_{1}, \cdots, r_{n}$ in $K$ such that $r_{1} s_{1}, \cdots, r_{n} s_{n}$ is a basis of $b S$; permuting the $s_{i}$, we see that $b S=r_{1} S$.

Corollary 1. Suppose $L$ is a Galois field extension of $K$ with group $G$, and assume that all the minimal primes of $R$ are unramified in $S$. Then $S$ is pseudo-Galois for $R[G]$, and hence

$$
\mathscr{P}(R, S) \cong H^{1}\left(G, S^{*}\right) \text {. }
$$

Proof. The fact that $S_{P}$ is Galois for $R_{P}[G]$ when there is no ramification is a well-known bit of folklore; much more general results are proved, e.g., in [2].

CoROLlaRY 2. Suppose L over $K$ is purely inseparable of degree $p$, and $D$ is a K-derivation with $D S \subseteq S$. Let $H=R[X]$ as above, and let $H_{0}$ be the image of $H$ in End $S$. Assume DS is not contained in any minimal prime of $S$. Then $S$ is pseudo-Galois for $H_{0}$, and hence

$$
\mathscr{P}(R, S) \cong \mathscr{Q}\left(H_{0}, S\right) \cong \mathscr{Q}(H, S)
$$

Proof. The hypotheses imply readily that $D^{p}=\lambda D$ for some $\lambda \in R$ [8, p. 63], and we have $H_{0} \cong R[X] /\left(X^{p}-\lambda X\right)$. Functions $h \mapsto b^{-1} h \cdot b$ are equal on $H$ if and only if they are equal on $H_{0}$, so the second isomorphism is trivial. To prove that $S$ is pseudo-Galois we may localize and assume that $R$ is a discrete valuation ring with maximal ideal $P$; by inseparability there is a unique maximal ideal $Q$ of $S$ lying over it. By hypothesis $S / P S$ has a nontrivial derivation $\bar{D}$ over $R / P$; in particular the two cannot be equal, and so $S / P S$ either is a $p$-dimensional field extension or has the form $(R / P)[Y] / Y^{p}$. In either case the hypothesis $D S \nsubseteq Q$ shows that $\bar{D} y$ is invertible for a generator $y$ of $S / P S$. If $D_{1}$ is the derivation with $D_{1} y=1$, we have $D_{1}=(1 / \bar{D} y) \bar{D}$ in the image of $H_{0} / P H_{0} \otimes S / P S$. But it is well known (and trivial) that $D_{1}$ and $S / P S$ generate End $S / P S$. Thus the map from $H_{0} / P H_{0} \otimes$ $S / P S$ is a surjection, and dimension count shows it is an isomorphism.

The isomorphism $\mathscr{P} \cong Q^{2}$ could be proved for these two cases by using the idea in Theorem 2, showing from the given hypotheses that an element $b$ with $h \cdot b \in b S$ comes locally from $R$. This is essentially 
what is done in [7]. But our argument brings out the general result underlying Samuel's two theorems. It also yields the extension to several derivations in [10, Th. 2.9]. In addition, the example in the next section shows that we can treat problems (with $L^{p} \nsubseteq K$ ) which cannot be handled by derivations.

3. The surface $Z^{q}=X Y$. Let $k$ be a field of positive characteristic $p$, and let $L$ be the fraction field of $S=k[x, y]$. Let $q$ be a power of $p$, and let $K$ be the fraction field of $R=k\left[x^{q}, y^{q}, x y\right]$. As in [8, p. 65], it is easy to see that $R=S \cap K$ and so is a Krull domain; it is the affine coordinate ring of $Z^{q}=X Y$ with $x^{q}=X$ and $y^{q}=Y$. Let $G$ be a cyclic group of order $q$, with generator $g$. Set $A=R[G]$ and map $S \rightarrow A \otimes_{R} S$ by $x \mapsto g \otimes x$ and $y \mapsto g^{-1} \otimes y$. Then the dual $H=R^{G}$ has a basis of idempotents $e_{0}, e_{1}, \cdots, e_{q-1}$ with $e_{\lambda} \cdot x^{i} y^{j}$ equal to $x^{i} y^{j}$ if $\lambda \equiv i-j(\bmod q)$ and equal to 0 otherwise. As an $R$-module, $S=\bigoplus e_{i} S$; the fixed ring is $e_{0} S=R$.

The map $S \otimes S=\bigoplus e_{i} S \otimes S \rightarrow A \otimes S$ takes $s_{i} \otimes t$ to $g^{i} \otimes s_{i} t$ for $s_{i} \in e_{i} S$. Thus to show that $S$ is pseudo-Galois we must show that the multiplication maps $e_{i} S \otimes S \rightarrow S$ are isomorphisms at each minimal prime $P$ of $R$. Since $L$ is purely inseparable over $K$, we know that $S_{P}$ is a local ring; the condition then is that $e_{i} S$ contain a unit of $S_{P}$, i.e., not lie in the maximal ideal. But obviously $e_{i} S$, which contains both $x^{i}$ and $y^{q-i}$, does not lie in any minimal ideal of $S=k[x, y]$. Hence $S$ is pseudo-Galois for $H$.

Take now an element $b$ with all $e_{i} b \in b S$; multiplying by an element of $K^{*}$, we may assume $b$ is a polynomial. Then $e_{i} b$ consists of some of its terms, and for all these to be multiples of $b$ requires that $b=$ $e_{i} b$ for some $i$. All such elements are $K$-multiples of $x^{i}$, and these give us a cyclic group of order $q$. Since $S$ has unique factorization, all divisors of $R$ become principal, and we have proved

Proposition 4. Let $k$ be a field of characteristic $p$, and $q$ a power of $p$. Then the divisor class group of $k\left[x^{q}, y^{q}, x y\right]$ is cyclic of order $q$.

We can carry out the same proof assuming only that $k$ is a unique factorization domain, just as was done in $[8, \mathrm{p} .65]$. (The result could be proved there, of course, only for $q=p$.)

4. Galois extensions and the kernel of Pic. Among the divisorial ideals of $R$ are the invertible ideals, and the group Pic $R$ of invertible ideals modulo principal ideals is a subgroup of the divisor class group. Thus the kernel of the map Pic $R \rightarrow \operatorname{Pic} S$ is a subgroup of $\mathscr{P}(R, S)$. In general it may well be smaller. In the example of $\S 3$, for instance, $\mathscr{P}(R, S)$ is generated by the inverse image of $x S$, 
which [4, p. 89] is just $x S \cap R$; this is not an invertible ideal. Suppose however that $S$ is flat over $R$. Then a divisorial ideal $D$ is mapped simply to $D S[4, \mathrm{p} .20]$; since $S$ is integral, it is faithfully flat over $R$, and so $D S$ principal implies $D$ invertible. Hence we have proved the following generalization of [10, Corollary 2.8]:

Proposition 5. Assume that $S$ is a pseudo-Galois H-module algebra and is flat over $R$. Then

$$
\mathscr{Q}(H, S) \cong \operatorname{Ker}(\operatorname{Pic} R \rightarrow \operatorname{Pic} S) \text {. }
$$

These hypotheses are true if $S$ is Galois for $H$. In fact, they nearly imply $S$ Galois, as the following theorem shows.

Theorem 3. Assume $S$ is a pseudo-Galois H-module algebra. The following are equivalent:

(1) $S$ is Galois for $H$.

(2) $S$ is a projective $R$-module.

Proof. By definition (1) implies (2), so assume (2). In the proof of Theorem 1 we saw that $S$ is an $R$-lattice; then $S \otimes S$ and $A \otimes S$ are projective $R$-lattices, and the map between them is an isomorphism at every minimal prime $P$.

To complete the proof we just recall that if $M$ is a projective $R$-lattice in a $K$-space $V$, then $M$ is finitely generated and $M=\bigcap M_{P}$. Since this result seems to have been omitted from [4], we sketch the proof. Writing $M$ as a direct summand of a free module gives us

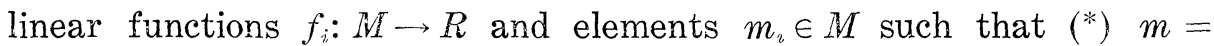
$\sum f_{2}(m) m_{i}$ for all $m \in M$. There is a natural extension of $f_{i}$ to a linear function $V \rightarrow K$, and $\left(^{*}\right)$ then holds for all $m \in V$. Let $v_{1}, \cdots, v_{n}$ be a basis of $V$, with dual basis $v_{i}^{*}, \cdots, v_{n}^{*}$, and write $f_{i}=\sum a_{\imath} v_{r}^{*}$. Applying $\left(^{*}\right)$ to the $v_{r}$ shows that $a_{i r}=0$ for all but finitely many $i$; thus $M$ is finitely generated. If $m \in \cap M_{P}$ then $f_{i}(m) \in \cap R_{P}=R$, so $m \in M$.

Corollary. Assume $R$ Noetherian, $S$ pserdo-Galois and flat. Then $S$ is Galois.

Proof. We have $S$ flat by hypothesis and finitely generated by the Porism to Theorem 1; hence $S$ is projective.

\section{REFERENCES}

1. S. A. Amitsur. Simple algebras and cohomology groups of arbitrary fieids, Trans. Amer. Math. Soc. 90 (1959), 73-112. 
2. M. Auslander and O. Goldman, The Brauer group of a commutative ring, Trans. Amer. Math. Soc. 97 (1960), 367-409.

3. N. Bourbaki, Algèbre Commutative, Ch. 5, 6, Hermann, Paris, 1964.

4. _ Algèbre Commutative, Ch. 7, Hermann, Paris, 1965.

5. S. U. Chase and M. E. Sweedler, Hopf Algebras and Galois Theory, ETH Springer Lecture Notes 97, Springer, New York, 1969.

6. A. Grothendieck, Technique de descente et théorèmes d'existence en géométrie algébrique, I. Sem. Bourbaki 190, 1959-1960, Benjamin, New York.

7. P. Samuel, Classes de diviseurs et dérivées logarithmiques, Topology 3 (1964), Suppl. $1,81-96$.

8. Lectures on Unique Factorization Domains, Tata Institute Lectures 30, Bombay, 1964.

9. M. E. Sweedler, Cohomology of algebras over Hopf algebras, Trans. Amer. Math. Soc. 133 (1968), 205-239.

10. S. Yuan, On logarithmic derivatives, Bull. Soc. Math. France 96 (1968), 41-52.

11. N. Zinn-Justin, Dérivations dans les corps et anneaux de charactéristique $p$, Bull. Soc. Math. France Mémoire 10, 1967.

Received December 16, 1969.

CORNELL UNIVERSITY 


\section{PACIFIC JOURNAL OF MATHEMATICS}

\section{EDITORS}

H. SAMELson

Stanford University

Stanford, California 94305

R. R. PHelPS

University of Washington

Seattle, Washington 98105
J. Dugundu

Department of Mathematics

University of Southern Californle

Los Angeles, California 9.0007

RICHARD ARENS

University of California

Los Angeles, California 9.0024

\section{ASSOCIATE EDITORS}
E. F. BECKENBACH
B. H. NeumanN
F. WOLE
K. Yoshida

\section{SUPPORTING INSTITUTIONS}

UNIVERSITY OF BRITISH COLUMBIA

CALIFORNIA INSTITUTE OF TECHNOLOGY

UNIVERSITY OF CALIFORNIA

MONTANA STATE UNIVERSITY

UNIVERSITY OF NEVADA

NEW MEXICO STATE UNIVERSITY

OREGON STATE UNIVERSITY

UNIVERSITY OF OREGON

OSAKA UNIVERSITY

UNIVERSITY OF SOUTHERN CALIFORNIA

\author{
STANFORD UNIVERSITY \\ UNIVERSITY OF TOKYO \\ UNIVERSITY OF UTAH \\ WASHINGTON STATE UNIVERSITY \\ UNIVERSITY OF WASHINGTON \\ AMERICAN MATHEMATICAL SOCIETY \\ CHEVRON RESEARCH CORPORATION \\ NAVAL WEAPONS CENTER
}

The Supporting Institutions listed above contribute to the cost of publication of this Journal, but they are not owners or publishers and have no responsibility for its content or policies.

Mathematical papers intended for publication in the Pacific Journal of Mathematics should be in typed form or offset-reproduced, (not dittoed), double spaced with large margins. Underline Greek letters in red, German in green, and script in blue. The first paragraph or two must be capable of being used separately as a synopsis of the entire paper. The editorial "we" must not be used in the synopsis, and items of the bibliography should not be cited there unless absolutely necessary, in which case they must be identified by author and Journal, rather than by item number. Manuscripts, in duplicate if possible, may be sent to any one of the four editors. Please classify according to the scheme of Math. Rev. Index to Vol. 39. All other communications to the editors should be addressed to the managing editor, Richard Arens, University of California, Los Angeles, California, 90024.

50 reprints are provided free for each article; additional copies may be obtained at cost in multiples of 50 .

The Pacific Journal of Mathematics is published monthly. Effective with Volume 16 the price per volume (3 numbers) is $\$ 8.00$; single issues, $\$ 3.00$. Special price for current issues to individual faculty members of supporting institutions and to individual members of the American Mathematical Society: $\$ 4.00$ per volume; single issues $\$ 1.50$. Back numbers are available.

Subscriptions, orders for back numbers, and changes of address should be sent to Pacific Journal of Mathematics, 103 Highland Boulevard, Berkeley, California, 94708.

PUBLISHED BY PACIFIC JOURNAL OF MATHEMATICS, A NON-PROFIT CORPORATION

Printed at Kokusai Bunken Insatsusha (International Academic Printing Co., Ltd.), 7-17, Fujimi 2-chome, Chiyoda-ku, Tokyo, Japan. 


\section{Pacific Journal of Mathematics}

\section{Vol. 36, No. 2 December, 1971}

George E. Andrews, On a partition problem of H. L. Alder ............ 279

Thomas Craig Brown, An interesting combinatorial method in the theory of locally finite semigroups .......................... 285

Yuen-Kwok Chan, A constructive proof of Sard's theorem ............. 291

Charles Vernon Coffman, Spectral theory of monotone Hammerstein

operators...................................... 303

Edward Dewey Davis, Regular sequences and minimal bases .......... 323

Israel (Yitzchak) Nathan Herstein and Lance W. Small, Regular elements in

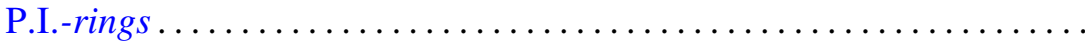

Marcel Herzog, Intersections of nilpotent Hall subgroups ..............

W. N. Hudson, Volterra transformations of the Wiener measure on the space

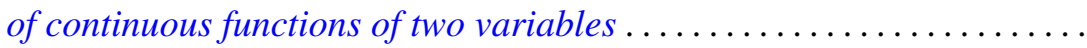

J. H. V. Hunt, An n-arc theorem for Peano spaces ................ 351

Arnold Joseph Insel, A decomposition theorem for topological group

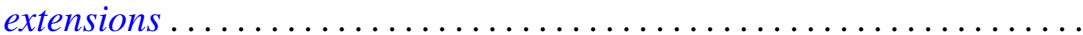

Caulton Lee Irwin, Inverting operators for singular boundary value

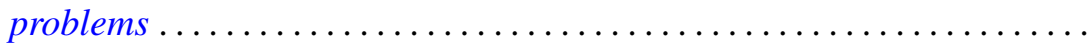

Abraham A. Klein, Matrix rings of finite degree of nilpotency ............ 387

Wei-Eihn Kuan, On the hyperplane section through a rational point of an algebraic variety...

John Hathway Lindsey, II, On a six-dimensional projective representation of $\mathrm{PSU}_{4}(3)$

Jorge Martinez, Approximation by archimedean lattice cones ...

J. F. McClendon, On stable fiber space obstructions .........

Mitsuru Nakai and Leo Sario, Behavior of Green lines at the Kuramochi boundary of a Riemann surface ....................

Donald Steven Passman, Linear identities in group rings. I. .

Donald Steven Passman, Linear identities in group rings. II ...

David S. Promislow, The Kakutani theorem for tensor products of

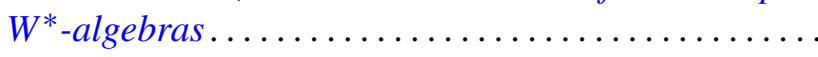

Richard Lewis Roth, On the conjugating representation of a finite group

Bert Alan Taylor, On weighted polynomial approximation of entire functions...

William Charles Waterhouse, Divisor classes in pseudo Galois

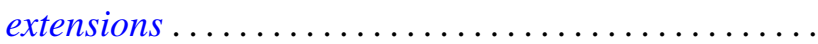

Chi Song Wong, Subadditive functions ...

Ta-Sun $\mathrm{Wu}$, A note on the minimality of certain bitransformation groups 